\title{
MODELAMIENTO DE LA ESTRUCTURA DEL ALUMINIO LIQUIDO MIEDIANTE EL METODO DE DINAMICA MOLECULAR
}

\author{
Justo A. Rojas Tapia, Chachi Rojas Ayala y Erich Manrique Castillo \\ Laboratorio de: Cristalogurfia Conapuracional, Facultad de Ciencias Físicas \\ Universidad Nacional Mayor de San Marcos

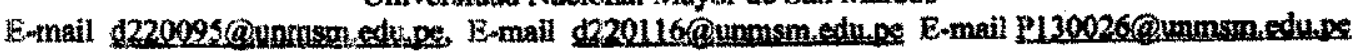

\begin{abstract}
AllSTRACT: We use the classical molecular dynamics method (MD) to obtain an atomistic description of the diffusion and the structure of liquid aluminum in a wide range of temperatures $(900 \mathrm{~K}-2600 \mathrm{~K})$. These studies use two-body long range oscillatory potentials. The numeric resolution of the motion equations is carried out by means of the Verlet algorithm. The $\mathrm{MD}$ simulation has been performed on a system of 256 particles using periodic boundary conditions to reduce finite size effects. The analysis of the distributions functions shows that short range order nanains up to high temperatures. The height and shape of the peaks suffer significant changes with the temperature, which evidences the peculiarities of changes in the liquid structure. The correlation radius decriasies from $0,9 \mathrm{~nm}$ to $0,4 \mathrm{~nm}$ in the range of temperatures studied. We found that the first coordingtion number changes approximately in the interval of $12-8.5$. The results of the measurements of the selfdiffusion coefficient in aluminum, carried out by means of the mean-squared displaciment of the ions, are in agreement with experimental data.
\end{abstract}

Kaywords: molecular dynarniss, simultion, aluminum, pair correlation, diffiusion.

SUMILLA: Usamos el método de Dinánica Molecular (DM) para obtener una descripción atomistica de los procesos de difusión y estructura del aluminio liquido en un ámplio rango de temperaturas ( $900 \mathrm{~K}-2600 \mathrm{~K}$ ). En el estudio se utilizo un potencial de interticción par oscilante de largo alcance. La solución numérica de las ecuaciones de movimiento se llevó acabo merliante el algoritmo de Verlet. La simulación computacional se realizo sobre un sistema de 256 partículas, aplicando condiciones de frontera periodicas con el fin de reducir los efiactos de superficie. El and́lisis de las funciones de distribución obtenidas muestran que la ordenación local se mantiene hasta altas tempexaturas. La forma y altura de los picos en las funciones de correlación par varian sifgnificativamente con la temperaiura, lo cual evidencra las peculiaridades de la variación de la estructura del liçuiclo. Establecemos que el radio de correlación disminuye desde 0,9 hasta $0,4 \mathrm{~nm}$ en el intervalo de temperaturas estudiado, mientras que el primer número de coordinación varia desde 12 hasta 8,5 aproxirnaclamente. El cceficiente de autodifusión del aluminio, determinado mediante el desplazamiento cuadrutico medio de las particulas, se encuentra en concotdancia con los datos experimentales.

Pulalaras Clavea: dinámica molectular, simulación, alumninio, correlación de pares, difusión

\section{H. INIRODUCCION}

La tecnologin modema de producción de las aleaciones es tal, que en determinado momento la ateación se encuentra en estado líquido. La estructura y constitución del material solidificado en gran medida depende de la prohistoria en estado liquido. Por ejemplo, ha sido establecido, que la preparación de la fundición y su "grado de inestabilitad influyen susten- cialmente sobre importantes propiedades en estado sóliclo tales como la plusticidad, resistencia y tenacidad.

A plesar de los grandes esfuerzcis y logros significativos en el estudio de lo constitución y propindudes de los liquidos en general, asi como de los metales y aleaciones amorfos ${ }^{i-4}$, el problema del modelo del liquido y las propiedades topológicas y geometricas de estructuras de empaquetamiento compacto sigue siendo actual, 5 . En este sentido los métodos de modelamiento computacional de la estructura de los liquidos ocupan un lugar muy importante, ya que son métodos únicos para estudiar los fenómenos a nivel atomico.

El método de Dinémica Molecular (DM), que permite estudiar la evolución detallada del sistema en el tiempo y el espacio ampliamente utilizado a partir del trabajo pionero de Alder et al.' con un potencial de esferas rigidas, hasta con potenciales de tipo Lennard Jones y otros mas realistas, en el estudio de los fluidos simples de elementos nobles y liquidos metálicos ${ }^{8-13}$. 
Desaforturadamente hay pocos trabajos relacionados con estudio de la estructura do los líquidos metálicos realizados en un amplio rango de temperaturas. Sin embargo, existen evidencias experimentales en difè- rentes sistemels metálicos ${ }^{14-16}$ que la estructura de la ordenación local varía con la temperatura de una forma no monótona y se conserva hasta altas temperaturas. En el presente trabajo usamos al método de DM para realizar un estudio detallado de la estructura del alumini is líquido como fiunción de la temperatura, centrando la atención en la obtención de las funciones de distribución.

\section{EL MODULO Y LAS MULACION COMPUTACIONAL.}

El método de DM consiste en lá integración numérica de las ecuaciones de movimiento para un sistema de N particulas que interactúan mediante cierto potencial $\varphi(r)$. La elección adecuada rel potencial de interacción atómica es primordial para el método de DM. Para el Aluminio el modelo del sistema que se asume es el de interacción par entre los iones del tnetal. El Harniltoniano del sistema ze da mediante la fórnula,

$$
\mathrm{H}=\sum_{i} \frac{p_{i}^{2}}{2 m}+\sum_{i} \sum_{j>i} \varphi\left(r_{i j}\right)
$$

donde $p_{j}$ es el momentum de la particula $j ; \varphi\left(x_{i j}\right)$ el potencial de interacción par, que depende solamente de la distancia entre las partículas $i$ y $j, y$ tiene la forma

$$
\varphi(r)=\frac{A}{r^{12}}-\frac{B}{r^{3}} \cos (2 k, r)
$$

donde A y B son ciertas constantes, $k_{f}$ - radio de la superficie de Fermi. El potencial es truncado a la distancia de $0,9 \mathrm{~nm}$.

La simulación se llevó acabo mediante la DM standard sobre un ensamble microcanónico (ETV) de 256 iones ubicados dentro de una celda cúbica de lado $L=1,68 \mathrm{~nm}$ (que corresponde a una densidad aprox mada de $2,37 \mathrm{~g} / \mathrm{cm}^{3}$ ). Además, con fines de ilustreción, consideramos un sistema bidinensional que consta de 100 partícules. Con el fin de minimizar los efectos de superficie y simular la rnuestra macrosicópica, se aplica la condición de frontera periódica, para to cual la celda central es rodeada por todos lados con 26 copias o imágenes idénticas, de tal manera que cada copia repite con exactitud los microestados de la celda central.

Inicialmente los iones son distribuidos en las cercanias de los nudos de una red fec.

Las velocidades iniciales de los iones se dan de acuerdo a la distribución de Maxwell a la temperatura requerida. La integración numérica de las ecuaciones de movimiento se realiza mediante el algoritmo de Verlet ${ }^{6,9}$. El paso de tiempo se elige de tal manera que sea aproximadamente en un orden menor que el periodo de oscilación de las partículas, es decir, $\Delta t=$ $10^{-14}$ a $10^{-15} \mathrm{seg}$. La estabilidad del sistem controla por la fluctuación de la energía total, la cual, en estado de equilibrio, no era mayor que $0.1 \%$. La temperatura del sistema se calcula a partir do la energía cinética promedio de las partículas. El equilibrio térmico para una determinada temperatura se logra luego de 200 a 400 pasos de tiempo. Las mediciones de las magnitudes macroscópicas se realizaron durante 1000 a 2000 pasos de tiempo.

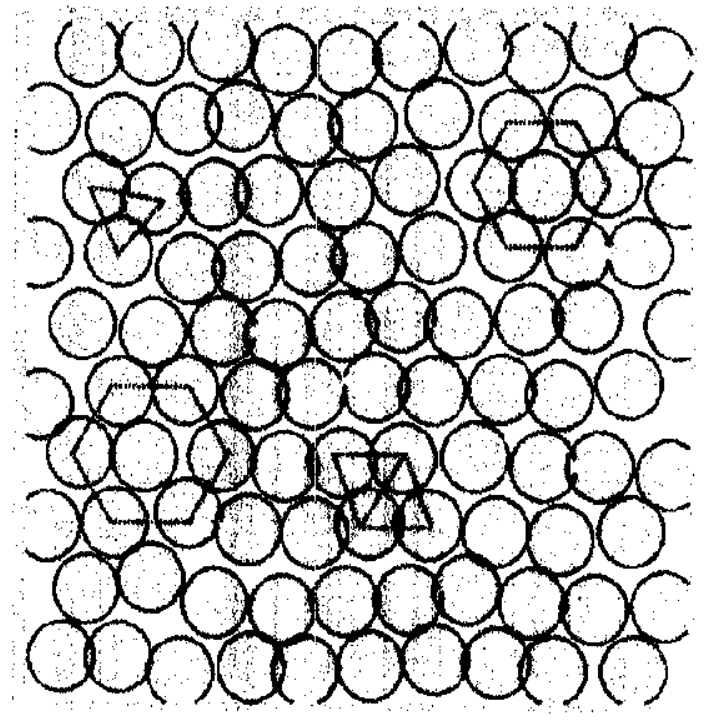

Figura 1. Posición instantánea de los iones de A! a $1100 \mathrm{~K}$. Los posibles clásteres están indicadas con lineas punteadas.

La función de correlación par, $g(t)$, la cual define la probabilidad de encontrar un par de átomos separados por una distancia $r$, respecto a lo esperado si la distribución fuera completamente caótica se determina mediante la relación 


$$
g(\mu)=\frac{1}{N \rho}\left\langle\sum_{i=1}^{N} \sum_{j \neq i}^{N} \delta\left(r^{\prime}-r_{i j}\right)\right\rangle
$$

donde $\rho$ es lia densidad media del sistema. Esta función es la principal fuente de información acerca de la estructura del material amorfo ${ }^{5,6}$.

\section{RESULTADOS Y DISCUSION}

En la Fugura Ise muestra la distribución espacial instantáasea tipica de los iones de Al en 2 aimertiones. El análisis de la evolución del sistema en el tiempo y a diferentes temperaturas, pirmite observar en forma cualitativa la estructura microheterogentea en forma de regiones nenometricas con diferentes estructuras de orden local dinámico (formisción de agregados o clasteres con estructura muy cercana a la cristalina). Es decir la estructura del liquido determinada tempera tura consta de una matrie desordenada y cierto número de diferentes agregados o clásteres.

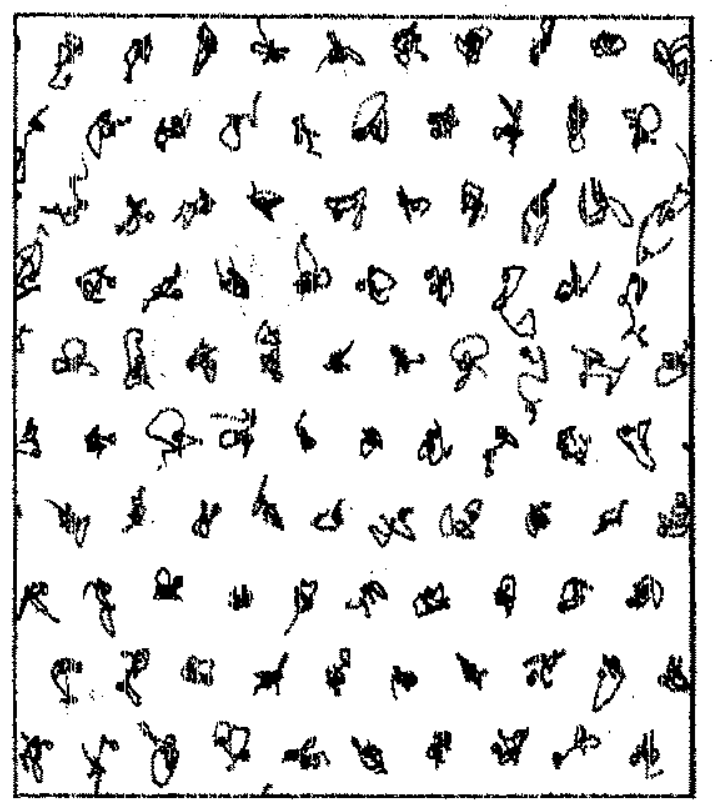

Algura 2. Troyecioria de 100 iones de Al a $1000 \mathrm{~K}$. Tisimpo de observación $t=0.5 \mathrm{ps}$.
En la Figura 2. Se muestra la evolución en el tiempo de la trayectoria de 100 particulas en el caso bidimensional. Como se puede apreciar de esta figura y del análisis de movimiento de los átomos en 3 dimensiones, concluimos que la gran mayoria de las particulas realizan un movimiento coordinado.

Algunos iones, durante todo el tiempo del experimento $(t * 1 \mathrm{ps})$ prácticamente no cambian su posición del centro de oscilación. Sin embargo, hay otras particulas, aunque pocas, que durante la mayor parte del tiempo participan en movimiento de deriva coordinado en grupo y luego, en un intervalo de tiempo muy pequetto realizan un salto a un nuevo centro de oscilación ( ver Figura 3).

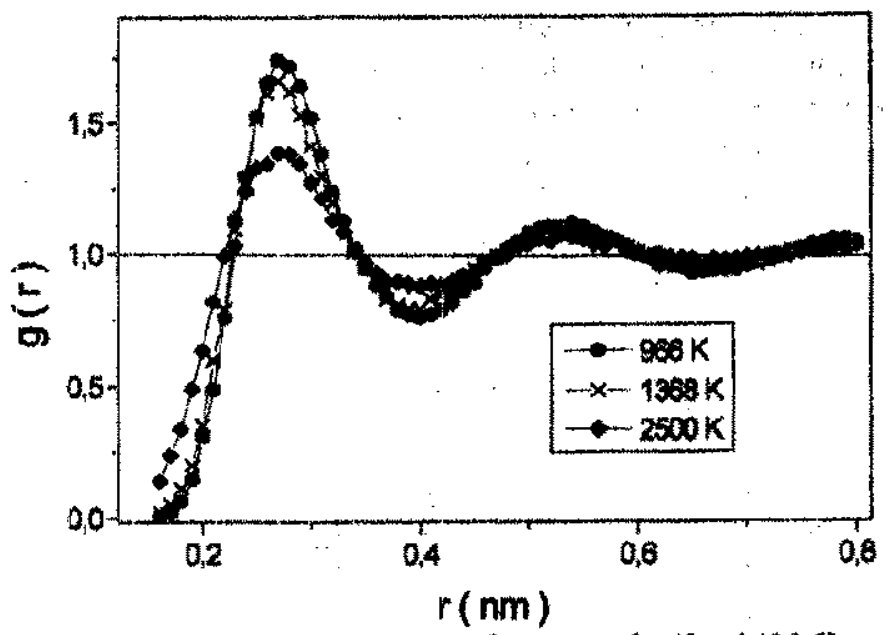

Figura 3. Trayectoria de un ion de Al a $1420 \mathrm{~K}$.

La función de correlación par $g(r)$, que es proporcional a la densidad local de distribución de los átomos, se ha deterninado en todo el intervalo de temperaturas estudiado y se muestra en la figura 4. para tres valores diferentes de la temperatura. La presencia de los máximos bien definidos nos indica que la distribución de los iones no es caótica. Ia probabilidad de encontrar un átomo alrededor de otro, tomado como origen, es máxima a una distancia aproximada de 0,28 nm. Esta distancia de máxima probabilidad no varía significativamente con la temperatura debido a que la simulación se realiza a yolumen constante.

Nosotros también hallamos las funciones de distribución radial $R(r)$, que caracteriza la cantidad de particulas dentro de una capa esferica con radio desde $r$ hasta $r+d r$. Estas funciones fueron determinadas a partir de las funciones de correlación par $g(\mathbf{r})$. 
Èn la Figura 5 mostramos una dependencia típica de $R(r)$ que corresponde a la temperatura $T=966 \mathrm{~K}$.

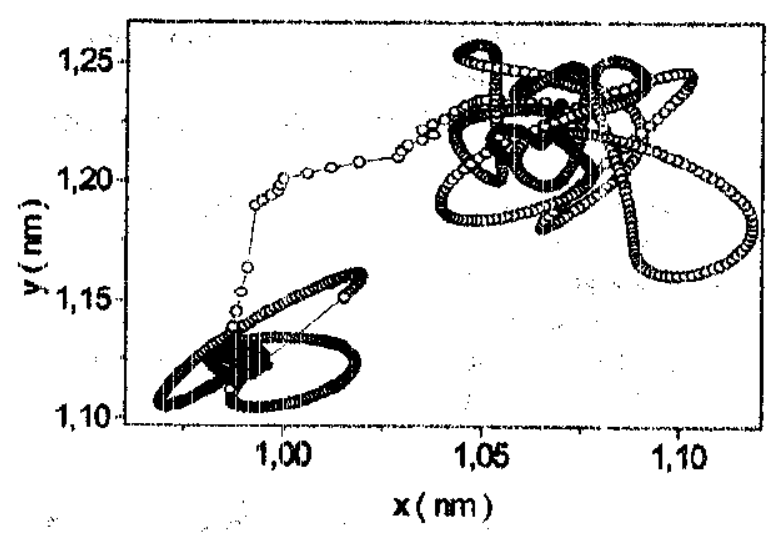

Figura 4. Función de correlación parl $g(r)$ del aluminio liquido. Tiempo de observación 5 ps.

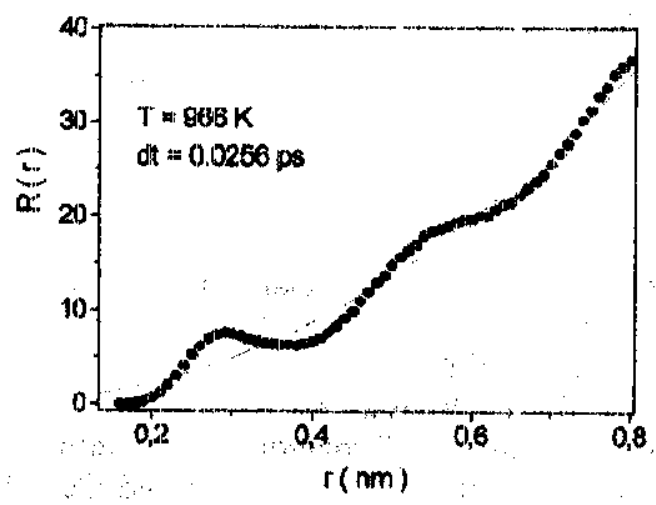

Figura S. Función de distribuctón racial del Aluminio liquido con $\Delta t=0.00 .5 \mathrm{ps}$.

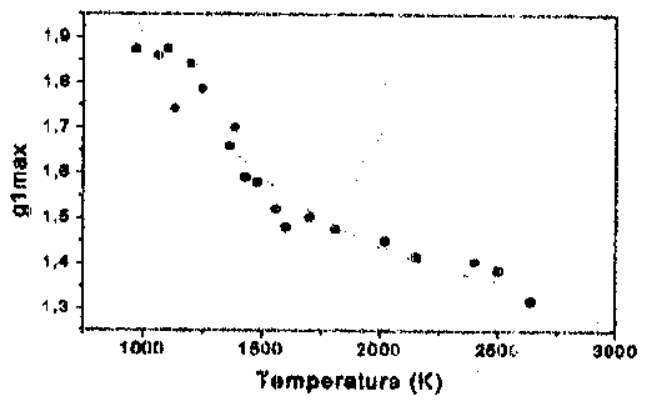

Nigura 6. Valor máximo del primer piso como función de la temperatura.
El análisis de las funciones de distribución a difenentes remperaturas muestra en primer lugar, que el grado de ordenación local disminuye con la temperatura, pero se conserva hasta altos valores de la misma; en segundo lugar, la forma y las características de los picos se diferencian significativamente, las culales reflejan las peculiaridades de la variación da la estructura del líquido, en particular, la ordenación local y estructura de los clásteres.

Además, se puede observar que la región de correlación abarca como máximo de 2 a 3 distar icias interatómicas.

Como se muestra en la Figura 6, la altura del primer pico o máximo en las funciones de distribución disminuye con la temperatura aproximadaments en forma exponencial.

El comportamiento de los otros picos es similar.

Esto significa que en el sistema estudiadr la distribución caótica de los átomos se logra a temperaturas mayores que $3000 \mathrm{~K}$.

A partir de la función de distribución radial $R(r)$ calculamos los números de coordinación $\mathrm{K}$ de las diferentes esferas evaluando el área debajo de los picos correspondientes, mediante la relación

$$
K_{1}=\int_{r_{1}}^{r_{2}} 4 \pi r^{2} g(r) d r
$$

El primer número de coordinación dismisuye aproximadamente de 12,5 a 8,5 en el intervalo de temperaturas estudiado. Estos resultados se encuentran en buena concordancia con los datos experimentales ${ }^{1,7}$.

El coeficiente de autodifusión del aluminio a temperatura $973 \mathrm{~K}$, determinado mediante el cál:ulo del Desplazamiento Cuadrático Medio (MSD) resulta ser igual $6,210^{-9} \mathrm{~m}^{2} / \mathrm{seg}$, el cual es cercano al valor experimental $5,410^{-9} \mathrm{~m}^{2} / \mathrm{seg}$. 


\section{CONCLIISIONES}

Apliçumos la técnica de DM para estudiar la estruatura del aluminio líquido usando un número relativanaente pequefio de átomos (debido a las limitaciones de la capacidad de memoria y velocidad de la PC usada). A pesar de estas lixuituciones se ha obtenido buenos resultados cuantitativos. Es de notar que la forma del potencial de interacción atómica es de vital importancia. En consecuencia ol uso de potenciales más realistas permite obtener resultados más exáctos. La estructura del liquiclo metálico, incluso de metales relativamente simples, como es el aluminio, es microheterogénea hasta altas temperaturas. En ella existen dinámicamente diferentes tipos de ordenación local, to, cual se manifiesta en las características de las funciones de distribución.

Mostarmos que el valor máximo de los picos en la funcibn $g(x)$ disminuye con la temperatura aproximadamente según una ley exponencial, y el radio de correlación abarca como máximo de 2 a 3 distancias interatónnicas. Los resultados obtenidos pueden servir como un aporte en el desarrollo de teorias brasadas en el los modelos de clásteres 1,4 .

Los datos obtenidos acerca de la función de correllación par pueden ser usados para determinar el factor de estructura $s(k)$ del aluminio, el cual se puede contrastar directamente con los datos experimentales de la difracción difusa de los rayos $\mathrm{X}$. Estos resultados serén reportados en un próximo trabajo, así como la descripción atomística detallacka de los procesos de difusión de los átomos.

\section{REFERENCLAS}

1. B. Baum. Liquidos metálicos. Problemas e hipótesis. Nauka, Moscú(1979).

2. D. Wilson. Estructura de los metales y aleaciones líquidas.

3. J.P. Hansen y I.R. McDonald. Theory of simple liquids, Academic Press(1986).

4. J. M. Ziman. Models of disorder. Cambridge University Press, Cambridge(1979).

5. M. Plischke y B. Bergersen. Equilibrium Statistical Physics. World Scientific, Singapure (1994).

6. M.P Allen y D. J. Tildesley. Computer Simulation of Liquids. Clarendon Press, Oxford (1987).

7. N.E Cusack, The Physics of strueturally disordered matter, lOP Publising Ltd (1987).

8. B.J.Alder and T.E. Wainwright, J.Chem. Phys. 27,1208 (1957).

9. L. Veriet, Phys.Rev, 165, 201 (1967).

10. Schiff Daniel. Computer "Experiments " on Liquid metals. Phys.Rev. 168,1 (1969).

11. G. Ciccottl, D.Frankel and I.R. McDonald, Simulations of liquids and solids, North Holland (1987).

12. 4,R. Ray, Tahir C. Fundamental treatment of molecular-dynamics ensembles. Phys. Rev. A,V37, N1 (1988).

13. Tahir C, Yoshitaka K. Molecular-dynamics simulations of glass formation and crystallization in binary liquid metals: $\mathrm{Cu}-\mathrm{Ag}$ and $\mathrm{Cu}-\mathrm{Ni}$. Phys. Rev. B, V59, N5 ( 1999).

14. Rojas Tapia J.A, Peculiaridades de la dependencia propiedad versus concentración de las soluciones metalicas homogéneas. Tesis de $\mathrm{Ph} . \mathrm{D}$, Jarkov (1985).

15. Харьков Е.И. Физика жидких металлов. Киев (1979).

16. Takeda S. Tamaki S. Local ordering features in the structure of liquid $\mathrm{Ga}-\mathrm{Te}$ alloys. J. Phys. Soc. Jap., V5, N6,(1983). 